\title{
The effects of rehearsing analyzed information upon the retrieval of unanalyzed information
}

\author{
MICHAEL T, TURVEY \\ THE OHIO STATE UNIVERSITY
}

Ss were required to recall unanalyzed information whilst in the process of remembering an analyzed sequence of items. Remembering the analyzed sequence by use of rehearsal did not reduce the availability of unanalyzed information. The data were discussed with respect to theoretical interpretations of rehearsal and a model was proposed emphasizing the separation of analyzed and unanalyzed information stores.

Unanalyzed information has been referred to by Melton (1963) as preperceptual information awaiting some form of categorization or encoding. This type of situation is manifested in the Sperling (1960) technique which involves the delayed partial sampling of S's retention of a tachistoscopically presented array of items. The limited duration of the array and uncertainty as to the dimension of the array to be recalled does not allow $S$ to categorize the information prior to the recall-cue. Based upon dichotic listening data Broadbent (1957, 1958) has proposed a distinction between two systems involved in the receiving and reproducing of information. The initial recipient, the S-system, is the store of raw sensory data awaiting encoding. For the purpose of this discussion, this system will be more commonly referred to as the preperceptual system. Subsequent to this is the P-system engaging in the analysis of input, capable of passing information only successively and in effect representing the ongoing perceptual activity. For Broadbent, the rehearsal of encoded information is interpreted as circulation of that information from the $\mathrm{S}$-system to the P-system and back again. This concept raises the question as to the effect of circulating analyzed information upon the efficiency of the preperceptual unanalyzed-information store. A number of authors (Conrad, 1962, 1964; Sperling, 1963; Wickelgren, 1965) have suggested that when a visual array of letters or digits is encoded, in terms of being translated into a verbal form, it is held in short-term store by means of an auditory code. Therefore it may be proposed that if such encoded information is circulating, i.e., being rehearsed, then it will not affect unanalyzed visual information since the former is represented as an auditory code and the latter is still a raw neural visual message. The present study probes this notion by using visual material, presented in the Sperling manner, interpolated between the encoding and recall of a sequence of items.

Method

The stimulus materials for the to-be-encoded information consisted of slides composed of a single row of five items. Three classes of items were used: A sequence of letters (HRYGX), a sequence of digits (94713), and a binary sequence (00101). The letter sequence was generated from items which were not present in the letter arrays for the Sperling procedure. The digit and binary sequences were randomly generated. The sequences were chosen on the basis of degree of similarity to the preperceptual information and their information content in terms of bits.

For the preperceptual information, $3 \times 5$ (three rows, five items) letter arrays were used, these being generated on the basis of no letter being repeated within a slide. A set of $3 \times 5$ digit arrays was employed in the training of the Ss.

The to-be-encoded information was displayed by means of a projector onto a dark pre-exposure field which was constantly projected by means of one channel of a two-channel tachistoscope. The other channel projected the preperceptual information for a duration of $100 \mathrm{msec}$. The initiation of the sequence of preperceptual information display and post-display cue was by means of a subject controlled switch. The post-display cue followed at a delay of $700 \mathrm{msec}$-a time interval chosen to enhance the possibility of interference from the encoded information. The post-display cue was one of three possible tones generated by an audio-oscillator and presented to the subject via earphones.

Ss were 100 male and female undergraduate university students enrolled in introductory psychology courses. The Ss were randomly assigned to the four conditions designated as encode-letters, encode-digits, encodebinary, and control. The control group was only exposed to the preperceptual slides. Initially each $\mathrm{S}$ was informed as to the nature of the preperceptual information displays. $S$ then received discrimination training for the three tones, the tones being adjusted for each $\mathrm{S}$ to obtain maximum ease of differentiation. The tones were labelled high, middle and low and were associated by $\mathrm{S}$ with the top line, middle line, and bottom line respectively of the information display. $S$ was then given training on the preperceptual task using $3 \times 5$ digit arrays. Twenty practice trials were given, with $S$ writing out the retrieved items on a response sheet. Following the training procedure, $S$ was then oriented to the nature of the experimental task. This consisted of a temporally controlled sequence of seven events. The first event, which was absent in the control condition, was the presentation of the to-be-encoded information. This slide's duration was dependent upon the length of time taken by $S$ to read it out loud once. With the termination of this slide, $S$ received a signal indicating that he had to press the switch in order to obtain the first (of five) preperceptual slides. At $10 \mathrm{sec}$. intervals, preceded by a ready signal, the preperceptual slides, consisting of 3 x 5 letter arrays, were presented. After the fifth 
preperceptual slide and a delay of 10 sec., a question mark appeared on the screen and $S$ attempted to recall the initially encoded information. $S$ recorded his retrieval following each presentation on an appropriate response sheet. The instructions given to $\mathrm{S}$ prior to the experimental sequence had stated that $S$ should try to do equally well on both tasks and should rehearse the encoded information between the preperceptual slide presentation in order to avoid forgetting.

Results

No significant difference in the amount of recall of unanalyzed information was obtained across the four conditions. Position in the sequence of slide presentations was significant $(F=11.07 ; d f=4 / 384 ; p<.001)$. However, since the interaction between conditions and position was nonsignificant, the significance of position is obviously not indigenous to the encoding conditions. Probably the position effect is due to changing familiarity with the letter arrays following training on the digit arrays.

Observation of the percentage error in the recall of the encoded information revealed $21.6 \%$ error for the letter condition, $3.2 \%$ error for the digit condition, and zero error for the binary condition. Analysis of variance showed this difference to be significant $(F=9.68$; $\mathrm{df}=$ $2 / 72 ; \mathrm{p}<.001)$.

\section{Discussion}

These data indicate that no matter what the nature and amount (in terms of bits) of recently encoded and rehearsed information, the amount of information retrieved from the visual preperceptual system (VPrS) is invariant. In effect, the circulation of analyzed information does not deplete the availability of unanalyzed information. That circulation occurred was inferred from the fact that the instructions suggested rehearsal; the nature of the information required rehearsal for retention and Ss in retrospect stated that they had rehearsed the initial information.

This conclusion is compatible with Broadbent's assumption of circulation between the $\mathrm{P}$ and $\mathrm{S}$ systems, since Broadbent argues that the S-system can pass information simultaneously and is not a limited capacity system. However, it is offered that this is not the case, i.e., the S-system, a store for unanalyzed information, is not utilized in rehearsal. This proposition is based on the following arguments. Firstly, Treisman (1964) has demonstrated differential qualities for analyzed and unanalyzed information and has indicated differential storage properties. Therefore, one might expect that once information had been analyzed it would be kept separate from the unanalyzed information. Secondly, Averbach \& Coriell (1961) and Steffy \& Eriksen (1964) in their studies suggest that information in the preperceptual store is easily erased by succeeding input. Thirdly, Conrad (1962, 1964), Sperling (1963) and Wickelgren (1965) have all profferred the notion that encoded information is held in short-term store by means of an auditory code. Therefore it would seem unlikely that raw, unanalyzed visual data and data framed in an acoustic code should intermingle in a single system. Such a concept is incompatible with our understanding of the parcellation of brain functions.

In view of these arguments the following model is presented to account for the data of the present experiment and similar data. When the initial information (the

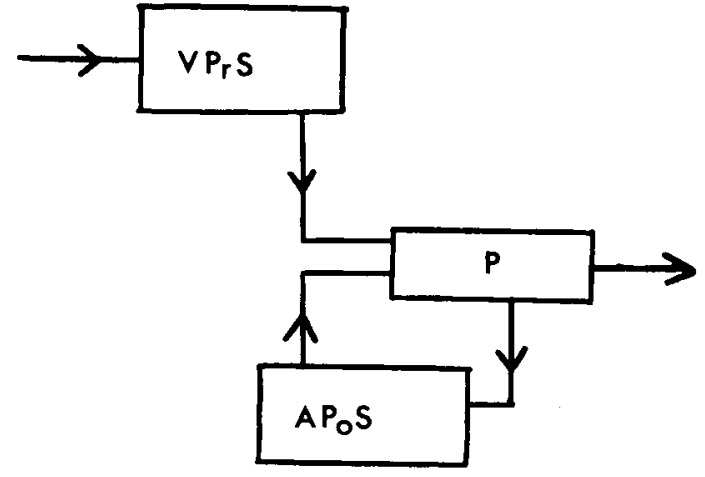

to-be-encoded information) is encoded by $S$ it is placed into an auditory postperceptual store (APoS). The process of rehearsal involves circulating this information between the APoS and a system (Broadbent's P-system) engaged in ongoing perceptual processes. Note how this differs from the Broadbent conceptualization in that the circulation now is between the P-system and a store for analyzed information. Further, it is observed that information retrieved from the VPrS does not pass through any other storage system and is therefore uninfluenced by other inputs. That $P$ is a processer and not a store is congruent with Broadbent's position. Also indicated by the model is the fact that when information is retrieved from the VPrS, it is transformed from raw visual data into a speech-motor code and is therefore able to enter the APOS. Now it can exert an influence upon the initially encoded information (as indicated by the considerable error occurring in the recall of the encoded letters).

\section{References}

Averbach, E., \& CorielI, A. S. Short-term memory in vision. Bell Syst. tech. J., 1961, 60, 309-328.

Broadbent, D. E. A mechanical model for human attention and immediate memory. Psychol. Rev., 1957, 64,205-215.

Broadbent, D. E. Perception and communication. New York: Pergamon Press, 1958.

Conrad, R. An association between memory errors and errors due to acoustic masking of speech. Nature, 1962, 193, 1314-1315.

Conrad, R. Acoustic confusions in immediate memory. Brit. J. Psychol., 1964, 55, 75-84.

Melton, A. W. Implications of short-term memory for a general theory of memory. J. verbal Learn. verbal Behav., 1963, 2, 1-21.

Sperling, G. The information available in brief visual presentations. Psychol. Monogr., 1960, 74 (Whole No. 498).

Sperling, G. A model for visual memory tasks. Hum. Factors, 1963, $5,19-36$.

Steffy, R. A., \& Eriksen, C. W. Short-term memory and retroactive interference in visual perception. J. exp. Psychol., 1964, 68, 423-434.

Treisman, A. Monitoring and storage of irrelevant messages in selective attention. J. verbal Learn. verbal Behav., 1964, 3, 449-459.

Wickelgren, W. A. Acoustic similarity and intrusion errors in shortterm memory. J. exp. Psychol., 1965, 70, 102-108. 